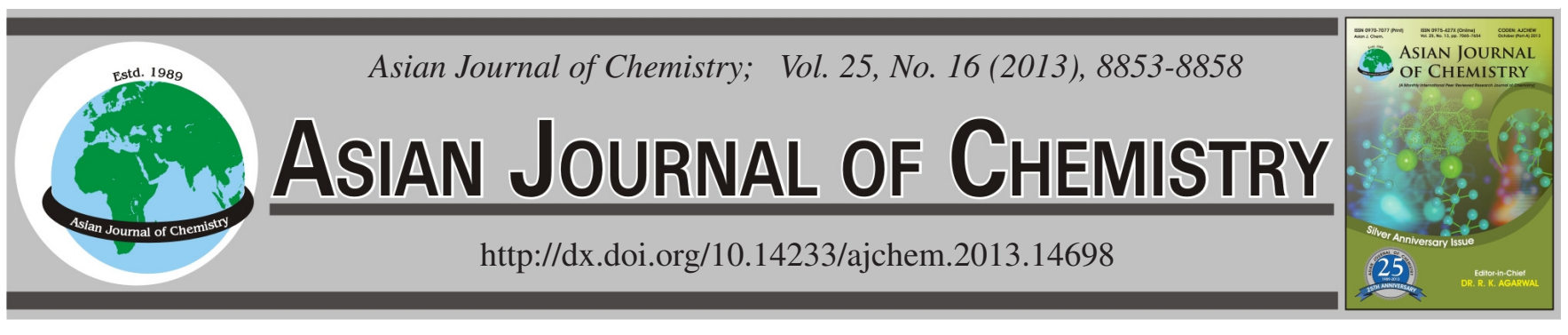

\title{
Evaluation of Aroma Components in Chinese Southwest Tobacco by Headspace Gas Chromatography-Mass Spectrometry
}

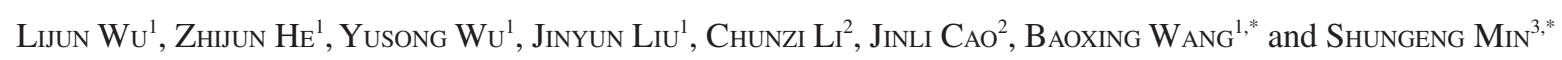

\author{
${ }^{1}$ Yunnan Academy of Tobacco Science, Kunming, P.R. China \\ ${ }^{2}$ Tobacco Corporation of Yunnan Dali, Dali, Yunnan Province, P.R. China \\ ${ }^{3}$ Department of Applied Chemistry, China Agricultural University, P.R. China \\ *Corresponding authors: Tel/Fax: +86 871 65389661; E-mail: 13508716519@163.com; minsg@263.net
}

In this paper, a simple method of headspace analysis (HSA) was applied to determine aroma components in Chinese southwest tobacco. Ninety-seven aroma compounds were extracted from tobacco under optimized experimental conditions. Meanwhile, it took $4 \mathrm{~h}$ for traditional method namely simultaneous distillation and extraction (SDE) for analysis, which is time-consuming and a waste of solvent. Compared with simultaneous distillation and extraction, headspace analysis is faster and more convenient in extracting tobacco aroma compounds. Moreover, six aroma compounds were first proposed in the research. The main aromatic components in tobacco detected by headspace can evaluate the tobacco quality and distinguish a specific variety from others. Headspace analysis method was proposed as an easy, rapid and environment-friendly method for the determination of aroma components in tobacco. The proposed method can not only enhance the extracting rate of aromas but meet the demand of qualitative analysis.

Key Words: Headspace, Simultaneous distillation and extraction, Aroma components, Tobacco, GC-MS.

\section{INTRODUCTION}

Tobacco is one of the most widely consumed commodities and the aroma components in tobacco are very important factors for evaluating the tobacco quality and the commercial value $^{1}$. Several thousands of compounds could be identified in tobacco and many of them contribute to the flavour, aroma and physiological effects which makes tobacco unique among other plants. Aroma components decide the quality and fragrance style of the tobacco ${ }^{2}$. It is necessary to establish a suitable sample preparation method and a sensitive analytical method to do research on the aroma compounds in tobacco. The traditional methods for isolating the flavour compounds from tobacco were using procedures such as solvent extraction, steam distillation ${ }^{4}$, simultaneous distillation and extraction $(\mathrm{SDE})^{5-7}$, supercritical fluid extraction ${ }^{8}$, solid-phase microextraction $^{9-11}$ and liquid phase microextraction ${ }^{12}$. These methods are in weak sensitivity due to the low concentration of target subjects in rather a sophisticated matrix. The process of this method is labor-intensive and time-consuming. For these studies, it is necessary to develop a rapid and convenient method to detect aroma components in tobacco. A different approach which is named as headspace (HS) analysis was proposed for extraction and determination of analytes ${ }^{13-17}$.
Tobacco is an extremely complex mixture of chemicals containing 6600 chemical constituents, 3800 components of which were originated in the tobacco leaf and another 2800 components were in the mainstream smoke. About one third of the chemical components are related to the aroma of tobacco.

Headspace analysis is a method that gives information on the composition of liquid and solid samples based on the results of the analysis of the contacting gas phase ${ }^{18}$. Headspace has been scarcely employed so far in spite of its potential usefulness in sample preparation ${ }^{19-22}$. The analysis of substances in this method are sampled and injected directly into the GC equipment in an on-line form, avoided loss and contamination. However, the headspace was firstly utilized to extract the aroma components in Chinese southwest tobacco in this research. The results suggested that this method can be used for rapid determination of the heavy aromas.

In present work, expecting for obtaining a simple, rapid and feasible method, we investigated the combination of headspace and GC-MS to determine total 97 aromatic constituents in tobacco samples. Compared with classical methods, headspace shows great advantages of simplicity, speed, low cost, automatic and no-pollution. It is significant to extracting aroma constituents from hongda rapidly. It is one of the most important planting type in Yunnan Province of China. 


\section{EXPERIMENTAL}

All reference substances were bought from Alfa Aesar with purity higher than $98 \%$ (Tianjin, China). Standard organic compounds including 5-methyl furfural, benzaldehyde, benzyl alcohol, 3-acetylpyridine, $\beta$-ionone and 2'-methylacetophenone were used for identification of the components of tobacco (Beijing, China). Nitrobenzene was used as an internal standard (Beijing, China). Dichloromethane (HPLC grade, Beijing, China) was selected as the extraction solvent in this work. Anhydrous sodium sulfate and sodium chloride were used for the simultaneous distillation and extraction (SDE) experiment and purchased from Kermel (Tianjin, China).

The Clarus 600 gas chromatograph (Perkin Elmer Corp., USA) was used to obtain chromatograms of the extract. The gas chromatograph was also equipped with an autosampler (Perkin Elmer Corp., USA) with capacity for 40 headspace vials composed of an oven for sample heating and a robotic arm where the headspace syringe was placed.

Sample preparation: One hundred thirty two tobacco samples were collected and grounded to powder, then passed through a 60-mesh sieve. The samples are from sampling points of Yunnan province: Xiangyun, Weishan, Yunlong, Binchuan, Nanjian, Heqing, Eryuan and Midu, respectively. The cultivars included hongda, K326 and yun87.

Simultaneous distillation and extraction: $15 \mathrm{~g}$ of tobacco powder was weighed and placed in a $1000 \mathrm{~mL}$ flask and 350 $\mathrm{mL}$ of saturated sodium chloride solution containing $0.03 \mathrm{mg}$ of nitrobenzene was added to this $1000 \mathrm{~mL}$ flask. $40 \mathrm{~mL}$ dichloromethane was placed in another $100 \mathrm{~mL}$ flask. Dichloromethane and the sample mixture were both boiled for $2 \mathrm{~h}$. After that, $200 \mathrm{~mL}$ of redistilled water and $10 \mathrm{~mL}$ of dichloromethane were distilled for $15 \mathrm{~min}$ to wash up the SDE system. Finally, about $50 \mathrm{~mL}$ of extract solution was obtained and dehydrated with $5 \mathrm{~g}$ of anhydrous sodium sulfate. It was reduced to $1 \mathrm{~mL}$ with a rotary evaporator at atmospheric pressure. Chromatographic separations were acquired on a HP$5 \mathrm{MS}$ column $(30 \mathrm{~m} \times 0.25 \mathrm{~mm}$, film thickness $0.25 \mu \mathrm{m})$. The injector temperature was set at $280{ }^{\circ} \mathrm{C}$. The column was held at $60{ }^{\circ} \mathrm{C}$ for $1 \mathrm{~min}$; subsequently, the temperature was raised to $135^{\circ} \mathrm{C}$ at $5{ }^{\circ} \mathrm{C} / \mathrm{min}$ then heated to $175^{\circ} \mathrm{C}$ at $2{ }^{\circ} \mathrm{C} / \mathrm{min}$, finally increasing to $250{ }^{\circ} \mathrm{C}$ at $5{ }^{\circ} \mathrm{C} / \mathrm{min}$ and held for $2 \mathrm{~min}$. Ultrahigh purity helium $(99.999 \%)$ was used as the carrier gas and the flow rate was $1.0 \mathrm{~mL} / \mathrm{min}$. Sample was injected using split mode with a split ratio of 10:1. The mass spectrometer was then operated as follows. The ionization voltage was set to $70 \mathrm{eV}$, the source temperature is $180{ }^{\circ} \mathrm{C}$ and the mass range is $m / z$ 50-350 amu.

Headspace analysis: The leaves of tobacco were cut into pieces and transfer $0.25 \mathrm{~g}$ of them to a $20 \mathrm{~mL}$ headspace vial then the vial was tightly sealed with crimp cap and white aluminum septa. Volatile substances were evaporated into the vial headspace and injected into the GC for further analysis. The operating conditions of the headspace were shown as below: incubation time, $40 \mathrm{~min}$; incubation temperature, 140 ${ }^{\circ} \mathrm{C}$; optimal pressurization time, $0.50 \mathrm{~min}$; injection time, 0.01 min. Chromatographic separations were acquired on a HP$5 \mathrm{MS}$ column $(15 \mathrm{~m} \times 0.25 \mathrm{~mm}, 0.25 \mu \mathrm{m}$ film thickness $)$. The column oven temperature was set at $60^{\circ} \mathrm{C}$ and then temperature heated to $200{ }^{\circ} \mathrm{C}$ at $15^{\circ} \mathrm{C} / \mathrm{min}$ holding for $1 \mathrm{~min}$. A constant column flow of $1.5 \mathrm{~mL} / \mathrm{min}$ of helium was used in this experiment. Injector and GC/MS transfer line temperatures were 280 and $250^{\circ} \mathrm{C}$, respectively. Sample was injected using split mode with a split ratio of 10:1. The mass spectrometer was then operated as follows. The ionization voltage was set to $70 \mathrm{eV}$, the source temperature to $180{ }^{\circ} \mathrm{C}$ and the mass range is $\mathrm{m} / \mathrm{z}$ 50-350 amu.

Data analysis: AMDIS software version 2.62 was used for the deconvolution and the NIST08 database was used for identification. Every sample was analyzed twice.

\section{RESULTS AND DISCUSSION}

Optimization of headspace conditions: In the headspace, the incubating temperature, the equilibration time and the pressurization time were discussed by a full factorial design $3^{3}$ that was performed to study the effects and the interactions of them. Incubating temperature at the stage of equilibrium was set from 80 to $120^{\circ} \mathrm{C}$, at intervals of $20^{\circ} \mathrm{C}$. Equilibration time was assessed from 20 to $60 \mathrm{~min}$, at an interval of $20 \mathrm{~min}$. Pressurization time was determined at time points from 0.5 to $1.5 \mathrm{~min}$, at an interval of $0.5 \mathrm{~min}$. The results were shown in Table-1. Injector and transfer line temperatures were selected to maintain vaporization conditions.

Based on comparison of peak areas and numbers that obtained from different experimental conditions, the subjects were chosen with good separation and reproducibility. The criterion of optimization was to select peaks as much as possible with the quantitative areas. The dominant factor for the experiment was determined by orthogonal experiment. Usually the results were analyzed by range analysis and variance analysis. The result of range analysis was listed in Table-1. According to the range of three variables, temperature has more infulence on the peak area and number than equilibrium time and pressurization time. The ideal condition was set at $120^{\circ} \mathrm{C}$, equilibrium time $40 \mathrm{~min}$ and pressurization time $0.5 \mathrm{~min}$. The result of variance analysis was shown in Tables 2 and 3. The temperature was the remarkable factor $(\mathrm{a}>0.05)$ and the optimum condition was the same as range analysis that was based on the value of F. Figs. 1 and 2 are the relationship of three variables between the peak area and the number respectively.

The peak area had significant changes accompanying with different temperatures. Moreover, peak area increased as temperature increasing. So it is necessary to have another experiment to research the suitable incubating temperature. With regard to incubating temperature, the response of peak area did not change when the temperature reached beyond $140{ }^{\circ} \mathrm{C}$ (Fig. 3). This temperature was high enough to collect massive volatile components and as the same time it avoided some unknown decomposition reactions at a higher temperature.

On the basis of these considerations, the conditions were selected as temperature $140{ }^{\circ} \mathrm{C}$, equilibration time $40 \mathrm{~min}$ and pressurization time $0.5 \mathrm{~min}$.

Selecting the length of column: The $30 \mathrm{~m}$ capillary column was applied in GC-MS generally. In this study, two chromatographic columns of $30 \mathrm{~m}$ and $15 \mathrm{~m}$ were checked. To separate the aromas in tobacco quickly and effectively, 
TABLE-1

$\mathrm{L}_{9}\left(3^{3}\right)$ TABLE OF THE ORTHOGONAL DESIGN AND THE RESULT OF PEAK AREA

\begin{tabular}{|c|c|c|c|c|c|}
\hline Experiment & Temperature $\left({ }^{\circ} \mathrm{C}\right)$ & $\begin{array}{l}\text { Equilibrium } \\
\text { time (min) }\end{array}$ & $\begin{array}{l}\text { Pressurization } \\
\text { time (min) }\end{array}$ & Peak area & Peak number \\
\hline 1 & 80 & 20 & 0.5 & 5375140 & 1 \\
\hline 2 & 80 & 40 & 1.0 & 8362321 & 1 \\
\hline 3 & 80 & 60 & 1.5 & 9790163 & 2 \\
\hline 4 & 100 & 20 & 1.0 & 10270598 & 1 \\
\hline 5 & 100 & 40 & 1.5 & 11242259 & 19 \\
\hline 6 & 100 & 60 & 0.5 & 12656448 & 27 \\
\hline 7 & 120 & 20 & 1.5 & 13564856 & 30 \\
\hline 8 & 120 & 40 & 0.5 & 16886005 & 36 \\
\hline 9 & 120120 & 60 & 1.0 & 13860916 & 26 \\
\hline Averange $^{1}$ (Area/No.) & $7842541 / 1$ & $9736865 / 11$ & $11639198 / 21$ & - & - \\
\hline Averange $^{2}$ (Area/No.) & $11389768 / 16$ & $12163528 / 19$ & $10831278 / 9$ & - & - \\
\hline Averange $^{3}$ (Area/No.) & $14770592 / 31$ & $12102509 / 18$ & $11532426 / 17$ & - & - \\
\hline Range & $6928051 / 15$ & $2365644 / 8$ & $106772 / 12$ & - & - \\
\hline
\end{tabular}

Averange $^{1}$, Averange $^{2}$, Averange ${ }^{3}$ means average of three levels of low, medium and high of peak area and number.

TABLE-2

RESULT OF VARIANCE ANALYSIS OF PEAK NUMBER

\begin{tabular}{|c|c|c|c|c|c|}
\hline Soruces of variation & DevSq & Degree of freedom & Variance & F-test & Significance level $(\alpha)$ \\
\hline Temperature & 1290 & 2 & 645.44 & 13.9976 & $0.0156^{*}$ \\
\hline Equilibrium time & 123 & 2 & 61.44 & 1.3325 & 0.3602 \\
\hline Pressurization time & 222 & 2 & 110.78 & 2.4024 & 0.2064 \\
\hline Revised deviation & 61.56 & 2 & 30.78 & 0.6675 & - \\
\hline Deviation & 184.44 & 4 & 46.11 & - & - \\
\hline Sum & 1700 & 8 & - & - & - \\
\hline
\end{tabular}

TABLE-3

RESULT OF VARIANCE ANALYSIS OF PEAK AREA

\begin{tabular}{lcccc}
\hline Soruces of variation & DevSq & Degree of freedom & Variance & F-test \\
\hline Temperature & $7.2011 \mathrm{e}+013$ & 2 & $3.6005 \mathrm{e}+013$ & 10.9181 \\
Equilibrium time & $1.1489 \mathrm{e}+013$ & 2 & $5.7443 \mathrm{e}+012$ & 0.7419 \\
Pressurization time & $1.1557 \mathrm{e}+012$ & 2 & $5.7787 \mathrm{e}+011$ & 0.2532 \\
Revised deviation & $7.1421 \mathrm{e}+012$ & 2 & $3.5710 \mathrm{e}+012$ & 0.1752 \\
Deviation & $1.9787 \mathrm{e}+013$ & 6 & $3.2978 \mathrm{e}+012$ & 1.0829 \\
Sum & $9.1797 \mathrm{e}+013$ & 8 & - & - \\
\hline
\end{tabular}
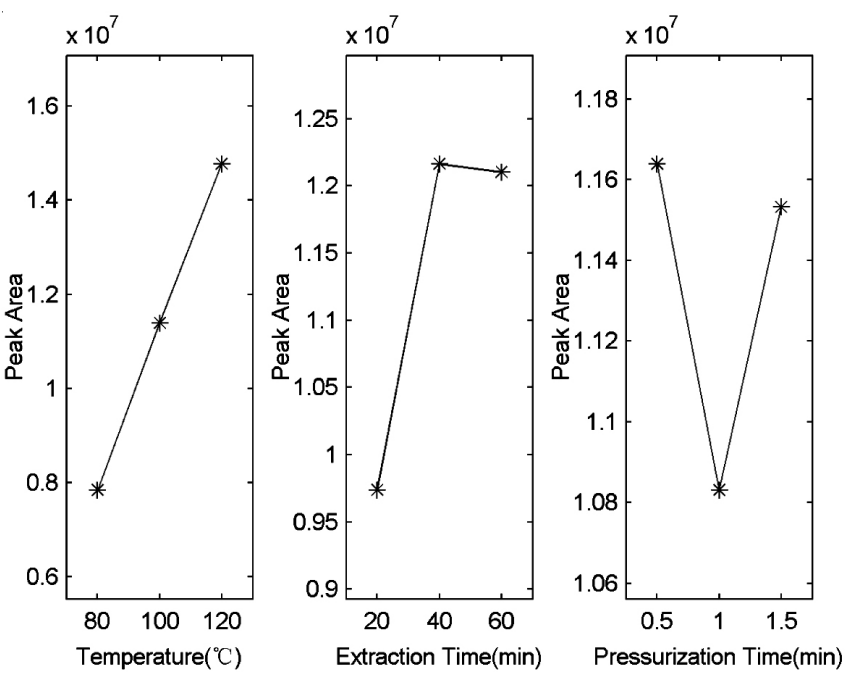

Fig. 1. Relationship between three variables and peak area

preliminary experiments were conducted to confirm the length of column. One hundred volatile components were identified with the longer column and 97 aromas were detected with the shorter one. The results showed that the result was almost
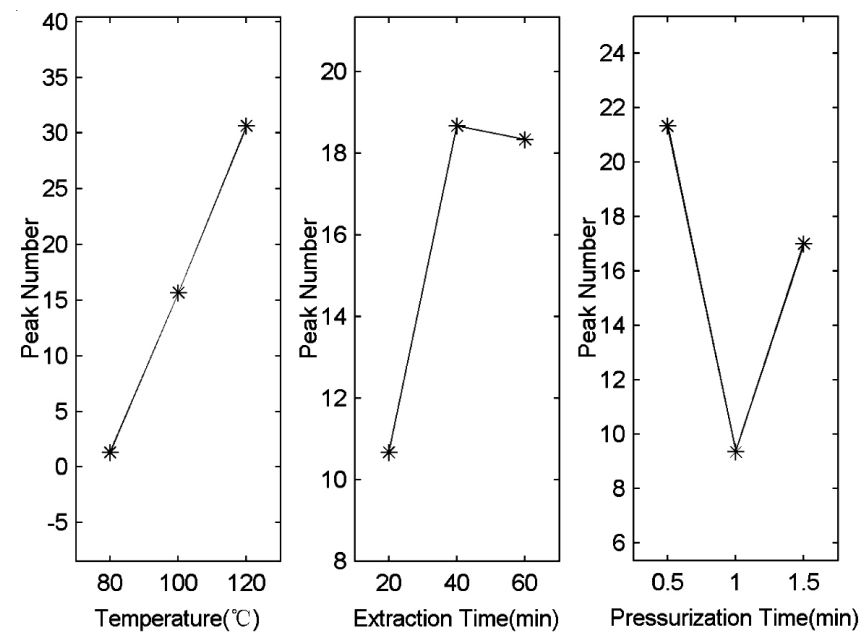

Fig. 2. Relationship between three variables and peak number

uniform in the condition of the two columns. However, they spent different time separating volatile components in tobacco. The shorter column just spent 11 min compared to the longer one $32 \mathrm{~min}$. So the $15 \mathrm{~m}$ capillary column was much more rapid and suitable for analyzing volatile components in tobacco. 


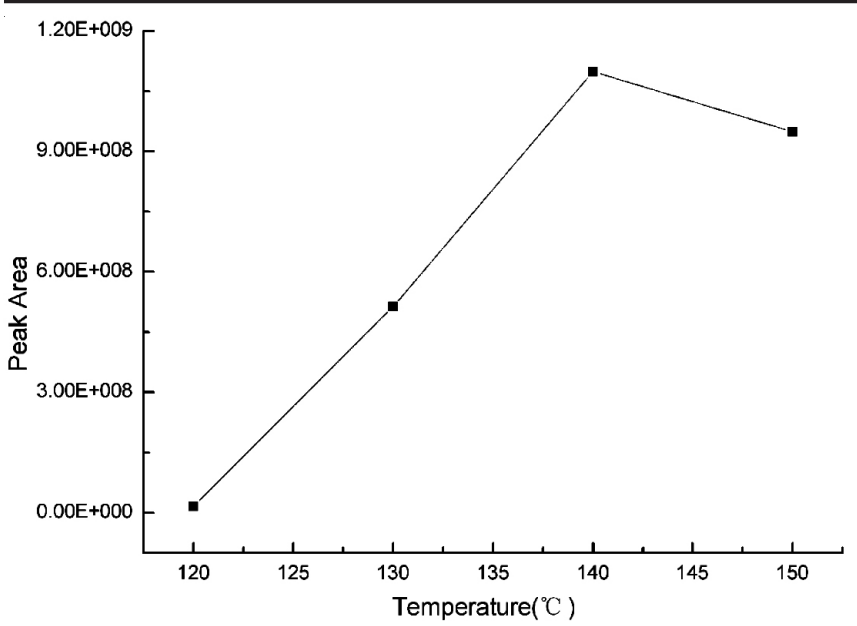

Fig. 3. Effect of heating temperature

Analysis performance: One hundred thirty two tobacco samples were analyzed by headspace and SDE, respectively. The target components were identified by the library searching with the high match values. Fig. 4 shows a total ion chromato- gram of headspace. The results are also listed in Table-4. Ninety seven aroma components among many separated peaks were verificated. The aroma substances such as furfuryl acetate, 1nonanal, decanal, 2-undecenal, isoquinoline and tetra decyl aldehyde were identified first by the method of headspaceGC-MS of compared with the method of SDE used in this paper and the other method of SD, SDE and HCD reported in the previous paper ${ }^{5,7}$.

In this paper, the method of SDE and headspace showed similar result for extracting and identifying the important aroma components in tobacco excepting the time on analytical process. Specifically, it took $4 \mathrm{~h}$ for SDE and $1 \mathrm{~h}$ for headspace. Headspace equipped with an autosampler and an oven which composed of 40 headspace vials for sample heating simultaneously save a lot of more time. The process of headspace is needless labors and poisonous solvent compared with SDE method. Components of aroma can be analyzed by chemometrics which calculated by abundance of characteristic ions. The important aromas such as 5-methyl furfural, benzyl alcohol, phenethyl alcohol and 6-methyl-5-hepten-2-one belong to sweet scent; $\beta$-ionone, 3 -acetylpyridine and $\beta$-damascenone

\begin{tabular}{|c|c|c|}
\hline \multicolumn{3}{|r|}{$\begin{array}{c}\text { TABLE-4 } \\
\text { RESULT OF HEADSPACE-GC-MS }\end{array}$} \\
\hline Retention time & CAS NO. & Name \\
\hline 1.137 & $1003-29-8$ & Pyrrole-2-carboxaldehyde \\
\hline 1.193 & $122-78-1$ & Phenylacetaldehyde \\
\hline 1.285 & $80-71-7$ & Methyl cyclopentenolone \\
\hline 1.307 & $100-52-7$ & Benzaldehyde \\
\hline 1.318 & $620-02-0$ & 5-Methyl furfural \\
\hline 1.345 & $3393-45-1$ & 5,6-Dihydro- $2 H$-pyran-2-one \\
\hline 1.349 & $100-51-6$ & Benzyl alcohol \\
\hline 1.379 & $533-37-9$ & Cyclopenta[b]pyridine \\
\hline 1.405 & $2399-48-6$ & Tetrahydrofurfuryl acrylate \\
\hline 1.444 & $110-93-0$ & 6-Methyl-5-hepten-2-one \\
\hline 1.457 & $1455-77-2$ & $1 H$-1,2,4-Triazole-3,5-diamine \\
\hline 1.487 & $623-17-6$ & Furfuryl acetate \\
\hline 1.499 & $142-82-5$ & Heptane \\
\hline 1.591 & $1072-83-9$ & 2-Acetyl pyrrole \\
\hline 1.680 & $92760-25-3$ & 2,3-Dehydro-1,8-cineole,1,3,3-trimethyl-2-oxabicyclo[2.2.2]oct-5-ene,dehydrocineole \\
\hline 1.705 & $765-69-5$ & 2-Methyl-1,3-cyclopentanedionea \\
\hline 1.713 & $823-82-5$ & Furan-2,5-dicarbaldehyde \\
\hline 1.735 & $97-88-1$ & Butyl methacrylate \\
\hline 1.755 & $3760-54-1$ & 1-Formylpyrrolidine \\
\hline 1.774 & $17678-19-2$ & 2-Furylhydroxymethylketone \\
\hline 1.792 & $3658-77-3$ & 2,5-Dimethyl-4-hydroxy-3(2H)-furanone \\
\hline 1.825 & $124-19-6$ & 1-Nonanal \\
\hline 1.899 & $60-12-8$ & Phenethyl alcohol \\
\hline 1.920 & 1072-83-9 & 2-Acetyl pyrrole \\
\hline 1.978 & $350-03-8$ & 3-Acetylpyridine \\
\hline 2.027 & $118-71-8$ & 3-Hydroxy-2-methyl-4H-pyran-4-one \\
\hline 2.088 & $23186-70-1$ & Allyl pentyl ether \\
\hline 2.093 & $3658-77-3$ & 4-Hydroxy-2,5-dimethyl-3(2H)-furanone \\
\hline 2.138 & $6950-04-5$ & 1,2-Di-(4-pyridyl)-1,2-dthanediol \\
\hline 2.172 & $29393-32-6$ & 5-Acetyltetrahydrofuran-2-one \\
\hline 2.176 & $1192-58-1$ & N-Methylpyrrole-2-carboxaldehyde \\
\hline 2.208 & $1125-21-9$ & 2,6,6-Trimethyl-2-cyclohexene-1,4-dione \\
\hline 2.264 & $93-60-7$ & Methyl nicotinate \\
\hline 2.453 & $28564-83-2$ & 2,3-Dihydro-3,5-dihydroxy-6-methyl-4( $H)$-pyran-4-one \\
\hline 2.660 & $13950-21-5$ & 1-Methyl-1H-pyrrole-2(5H)-one \\
\hline 2.742 & $112-26-7$ & 1,3-Cyclohexadiene-1-carboxaldehyde, 2,6,6-trimethyl- \\
\hline 2.825 & $112-31-2$ & Decanal \\
\hline 2.851 & $32780-06-6$ & (S)-5-Hydroxymethyldihydrofuran-2-one \\
\hline
\end{tabular}




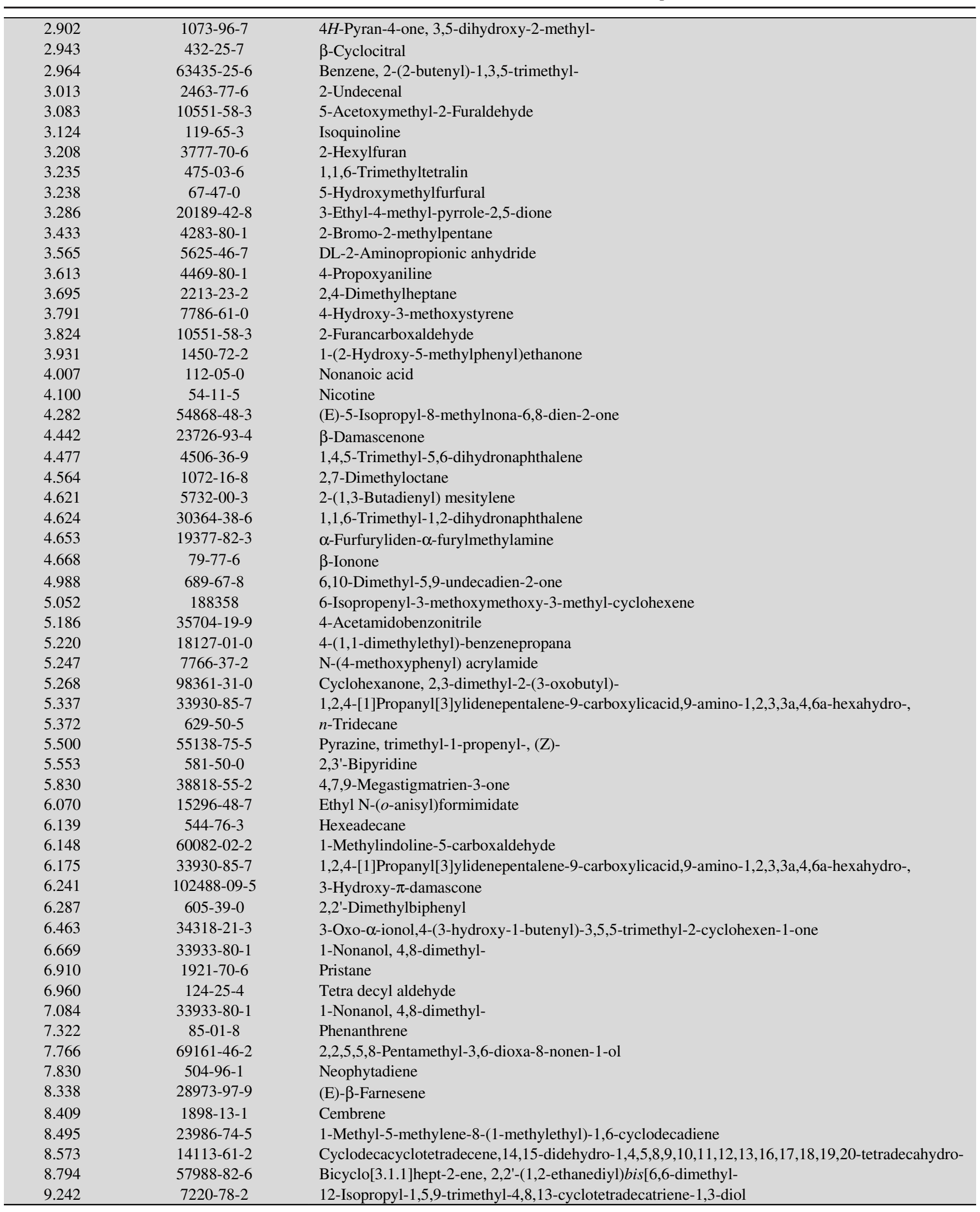

belong to faint scent. Complying with the comparison of flavour style and content from different aromas, the fragrance and sweet content of yun87 and K326 were inferior to hongda. Hongda possesses fresh and grace full aroma, representing the light pure aroma of tobacco obviously. It is meaningful to use rapid extraction for aroma constituents to evaluate the quality and distinguish different varieties from large amount samples.

\section{Conclusion}

An analytical method utilizing headspace-GC-MS was firstly developed for the analysis of volatile compounds in 


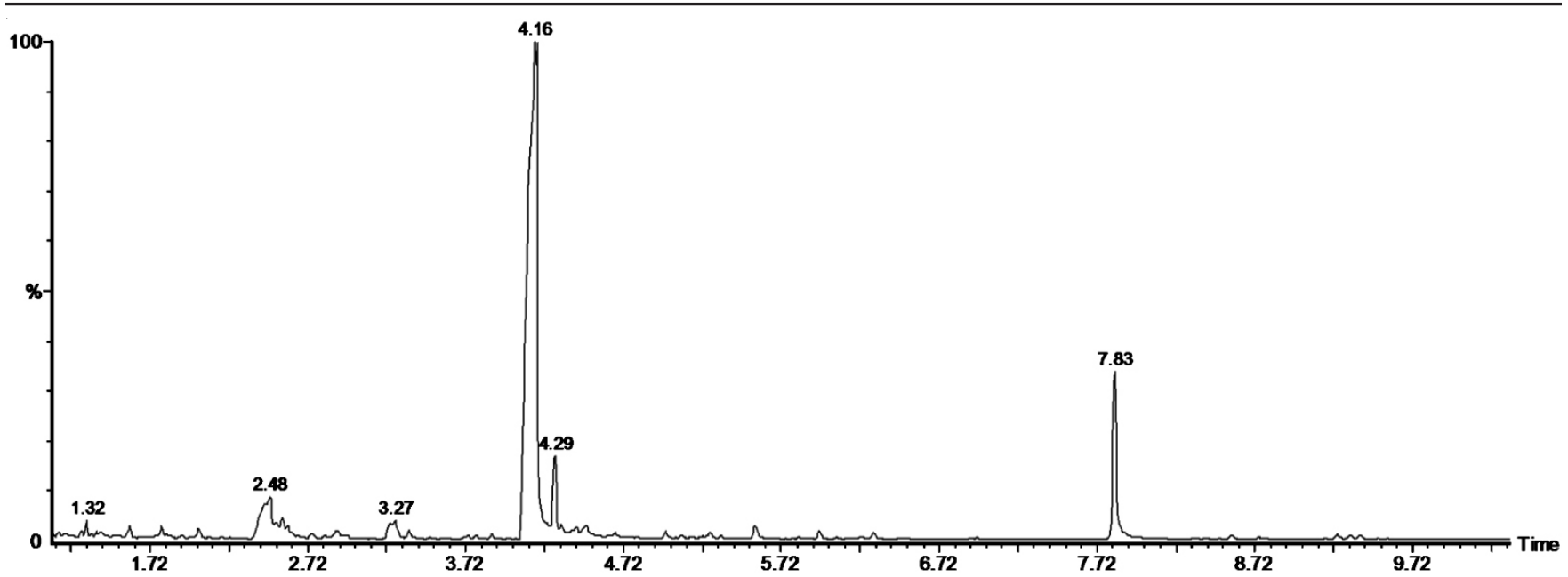

Fig. 4. Total ion chromatogram of tobacco

tobacco from different regions and cultivars in Chinese southwest tobacco. Ninety seven volatile components were identified and 6 of them were firstly identified by the method of headspace-GC-MS. The analysis took short time (about 11 min) to separate volatile components without sample pretreatment, clean-up stages, expensive materials and extensive laboratory works. Rapid extraction for aroma constituents of hongda as the most important planting type in Yunnan province of china, as well as distinguishing different varieties and the evaluation of tobacco quality, has a great significance. Nosolvent, rapidly, easy pretreatment, environmentally friendliness and automatically will make this approach very promising.

\section{ACKNOWLEDGEMENTS}

This work supported by the project of the Tobacco Corporation of Yunnan Dali (No. 09YN016).

\section{REFERENCES}

1. C.F. Merckel, A. Pragst, Ratzinger, B. Aebi, W. Bernhard and F. Sporkert, J. Chromatogr. A, 1116, 10 (2006).

2. L.F. Huang, M.J. Wu, X.J. Sun, K.J. Zhong, Z.M. Guo, Y.H. Dai, K.L. Huang and F.Q. Guo, J. Cent. South Univ. Technol., 4, 504 (2007).

3. K.J. Sun, M.L. Wang, Y. Shen and B.Z. Liu, Chinese Tobacco Science, 28, 23 (2007).

4. L.G. Zhang, C. Zhang, L.J. Ni, Y.J. Yang and C.M. Wang, Sep. Purif. Technol., 77, 261 (2011)
5. F.M. Peng, L.Q. Sheng, B.Z. Liu, H.W. Tong and S.M. Liu, J. Chromatogr. A, 1040, 1 (2004).

6. S. Selli and G. Cayhan, Microchemical J., 93, 232 (2009).

7. L.F. Huang, K.J. Zhong, X.J. Sun, M.J. Wu, K.L. Huang, Y.W. Li, Anal. Chim. Acta, 575, 236 (2006).

8. L.H. Wang, Y.H. Mei, F. Wang, X.S. Liu and Y. Chen, Sep. Purif. Technol., 77, 397 (2011).

9. S.S. Yang, C.B. Huang and I. Smetena, J. Chromatogr. A, 942, 33 (2002).

10. S. Bogusz Jr, A. de Marchi, T. de Melo, C.A. Zini and H.T. Godoy, J. Chromatogr. A, 1218, 3345 (2011).

11. M. Cirlini, A. Caligiani, L. Palla and G. Palla, Food Chem., 124, 1678 (2011).

12. S.H. Sun, Z.H. Cheng and J.P. Xie, Rapid Commun. Mass Spectrom., 19, 1025 (2005).

13. W. Thomas and P.L. Erns, J. Sep. Sci., 24, 885 (2001).

14. C.H. Deng, N. Yao, N. Li and X.M. Zhang, J. Sep. Sci., 28, 2301 (2005).

15. L.M. Wu, X.M.Shen and Q. David, J. Pharm. Biomed. Anal., 48, 8 (2008).

16. R.M. Cavalcante, M.V.F. de Andrade, R.V. Marins and L.D.M. Oliveira, Microchem. J., 96, 337 (2010).

17. G.L. Bienvenida, R.M. José, F. Juan, R. Garc and M.D. Antonio, Talanta, 83, 391 (2010).

18. A.G. Vitenberg, J. Anal. Chem., 58, 2 (2003).

19. A. Stambouli, A. El Bouri, T. Bouayoun and M.A. Bellimam, Forensic Sci. Int., 146S, S191 (2004).

20. M.M.H. Huisman, A. Oosterveld and H.A. Schols, Food Hydrocolloids, 18, 665 (2004).

21. C.M. Armanino, C. Casolino, M. Casale and M. Forina, Anal. Chim. Acta, 614, 134 (2008).

22. S. Maria, M. Bernardo, N. Goncalves, R. Lapa, B. Barbosa, F. Mendes and I.G. Pinto, Talanta, 80, 104 (2009). 